\title{
Qualidade de vida de estudantes de pós-graduação em Odontologia: uma análise por meio dos domínios do WHOQOL-bref
}

\author{
Cinthia Nara Gadelha Teixeira*; Maria Imaculada de Queiroz Rodrigues**; Paulo Goberlânio de \\ Barros Silva***; Myrna Maria Arcanjo Frota Barros***; Cláudia Maria Coêlho Alves****; Maria \\ Eneide Leitão de Almeida*****
}

\author{
* Doutora em Odontologia, Universidade Federal do \\ Maranhão \\ ** Mestranda em Clínica Odontológica, Universidade Federal \\ do Ceará \\ *** Doutor(a) em Clínica Odontológica, Universidade Federal \\ do Ceará \\ **** Professora Titular, Programa de Pós-Graduação em \\ Odontologia, Universidade Federal do Maranhão \\ ****** Professora, Programa de Pós-Graduação em Odontologia, \\ Universidade Federal do Ceará
}

Recebido: 05/06/2020. Aprovado: 18/11/2020.

\begin{abstract}
RESUMO
O objetivo do estudo foi avaliar a qualidade de vida (QV) dos estudantes de pós-graduação em Odontologia por meio dos domínios do World Health Organization Questionnaire for Quality of Lifebref (WHOQOL-bref). Trata-se de um estudo transversal, com 88 estudantes matriculados em um programa de pós-graduação em Odontologia. Os dados foram coletados entre junho e agosto de 2015, incluindo informações sociodemográficas e um questionário de qualidade de vida, o WHOQOL-bref. Foi realizada associação das informações sociodemográficas com os domínios do questionário utilizado e análise bivariada, seguida do modelo de regressão logística multinominal, sendo considerado significativo $\mathrm{p}<0,05$. A maioria $(55,7 \%)$ dos estudantes foram classificados na categoria "satisfação" quanto à qualidade de vida. Entre os domínios, o maior escore encontrado foi para Relações Sociais $(15,23 \pm 2,72)$, e o menor para o Físico $(12,82 \pm 1,56)$. Houve associação da frequência da prática religiosa ( $\mathrm{p}=0,046)$ e do ano da pós-graduação $(\mathrm{p}=0,025)$ com o domínio Físico; o sexo $(\mathrm{p}=0,016)$ e considerar-se religioso $(\mathrm{p}=0,008)$ com o domínio Psicológico; e o estado civil com os domínios Relações Sociais $(\mathrm{p}=0,017)$ e Meio Ambiente $(\mathrm{p}=0,008)$. A QV dos estudantes de pósgraduação em Odontologia foi mensurada na região de satisfação, podendo ser influenciada por fatores como religiosidade, ano da pós-graduação, sexo e estado civil.

Descritores: Qualidade de Vida. Odontologia. Educação de Pós-graduação em Odontologia.
\end{abstract}

\section{INTRODUÇÃO}

Qualidade de vida (QV) é definida como a percepção que o indivíduo tem da sua posição na vida, no contexto da cultura, e do sistema de 
valores em que vive e em relação aos seus objetivos, expectativas, padrões e preocupações ${ }^{1}$. Um comprometimento direto na QV dos indivíduos, pode impactar em problemas reais no cotidiano, refletindo, inclusive, na saúde mental.

A saúde mental da população universitária tornou-se foco de estudos ${ }^{2,3}$, pois ela se depara com desafios e mudanças no estilo de vida, como separação da família e dos amigos, maior independência e responsabilidades, podendo levar à exposição de diversos riscos à saúde ${ }^{4}$. Com os discentes de pós-graduação não é diferente.

Muitas vezes a QV dos estudantes do âmbito acadêmico é afetada, em especial os da pósgraduação stricto sensu, cursos caracterizados por exigências diferenciadas, como um extenso volume de publicação ${ }^{5}$, a ausência de tempo, tanto para estudo, como para a vida particular ${ }^{6}$, além da incerteza quanto à futura inserção profissional ${ }^{7}$.

Um levantamento realizado pelo "Plano de Ação em Ciência, Tecnologia e Inovação: Principais Resultados e Avanços", do Ministério da Ciência e Tecnologia ${ }^{8}$, destacou um notável crescimento do sistema de pós-graduação stricto sensu no Brasil. Porém, talvez como um efeito indesejado dessa escalada, o avanço da pósgraduação no país trouxe consequências notáveis, em especial para os estudantes, tendo sido caracterizado por uma elevada pressão ${ }^{6}$.

Nesse contexto, pesquisas envolvendo a QV em alunos de pós-graduação têm sido mais frequentes na literatura internacional. Pesquisas com esse público já investigaram o bem-estar psicológico ${ }^{9}$; as diferentes estratégias para o enfrentamento do estresse ${ }^{10}$; a qualidade de vida relacionada à saúde, onde o estresse percebido e o aculturativo impactaram negativamente ${ }^{11}$ na $\mathrm{QV}$ destes estudantes. Uma outra pesquisa mostrou também que variáveis como o sexo, estado civil e ano da pós-graduação impactaram diretamente na $\mathrm{QV}^{12}$.

No Brasil, estudo envolvendo a QV dos estudantes de graduação e pós-graduação em Odontologia de São Paulo permitiu a detecção precoce das dificuldades vivenciadas pelos estudantes, auxiliando no delineamento de estratégias que beneficiaram a busca por soluções para os conflitos que incidiam na $\mathrm{QV}^{13}$. Uma outra pesquisa com estudantes de pós-graduação em Odontologia no Ceará avaliou o efeito das atividades de educação a distância implantadas devido ao isolamento social na pandemia de COVID-19, sendo o domínio Psicológico o mais afetado $^{14}$.

Contudo, estudos que analisaram a QV de estudantes de pós-graduação por meio do World Health Organization Questionnaire for Quality of Life-bref (WHOQOL-bref), analisando os domínios desse instrumento em relação aos fatores sociodemográficos dos estudantes, não têm sido tão evidentes. O WHOQOL-bref foi o instrumento escolhido por demonstrar boa consistência interna, validade discriminante, concorrente e de conteúdo, e confiabilidade teste-reteste ${ }^{15}$. Deste modo, justifica-se a necessidade de realização deste estudo, cujo objetivo foi avaliar a qualidade de vida dos estudantes de pós-graduação em Odontologia por meio dos domínios do WHOQOL-bref.

\section{METODOLOGIA}

Foi realizado um estudo observacional, do tipo transversal, e quantitativo com os estudantes do Programa de Pós-graduação stricto sensu em Odontologia da Universidade Federal do Ceará (PPGO/UFC). Estudos transversais são muito empregados em investigações epidemiológicas, pois são de baixo custo, possuem simplicidade analítica, além de alto poder descritivo e simplicidade na coleta de dados ${ }^{16}$.

O PPGO/UFC fica localizado em Fortaleza, capital do estado do Ceará, Brasil. A coleta de dados aconteceu entre junho e agosto de 2015, nas salas de aula, laboratórios e clínicas do PPGO/UFC. Os dados foram coletados por três 
pesquisadoras, sendo descartado o treinamento destas por ser o instrumento utilizado de fácil entendimento, aliado ao alto nível intelectual dos pesquisados.

Os critérios de inclusão englobaram os estudantes regularmente matriculados no PPGO/UFC em 2015, enquanto os de exclusão envolveram aqueles alunos que estariam afastados do PPGO por licença saúde, com matrículas trancadas ou estudando fora do país. $\mathrm{O}$ universo dos estudantes foi constituído de 88 alunos, incluindo mestrandos, doutorandos e pósdoutorandos. Esse número coincidiu com a amostra, pois todos participaram do estudo, justificando a não realização do cálculo amostral.

O caderno de pesquisa continha dados sociodemográficos dos participantes, como sexo, estado civil, se possuíam filhos, trabalho, renda mensal, questões quanto à religiosidade, bolsa de estudos, curso e ano na pós-graduação, além do WHOQOL-bref, instrumento de mensuração de $\mathrm{QV}$, autorrelatado, transcultural, traduzido $\mathrm{e}$ validado no Brasil ${ }^{15}$.

Para o preenchimento do WHOQOL-bref, o entrevistado deveria considerar os quinze dias anteriores à aplicação do instrumento. Ele contém 26 questões, cada uma delas com cinco opções de respostas em escala do tipo Likert, que variam de 1 (nada/muito ruim/nunca) a 5 (extremamente/ completamente/muito bom/sempre). As duas primeiras questões são gerais, enquanto as demais encontram-se distribuídas em quatro domínios: Físico, Psicológico, Meio Ambiente e Relações Sociais ${ }^{17}$.

O domínio Físico é composto por sete questões, que envolvem quesitos sobre dor e desconforto, energia e fadiga, sono e repouso, mobilidade, atividades da vida cotidiana, uso de medicamentos e capacidade para o trabalho. $\mathrm{O}$ domínio Psicológico engloba seis questões, que tratam sobre sentimentos positivos e negativos, pensar e aprender, memória e concentração, imagem corporal e espiritualidade. O domínio Relações Sociais é formado por três questões, que abrangem relações pessoais, suporte social e atividade sexual. O domínio Meio Ambiente compreende oito questões, que incluem a segurança física e proteção, o ambiente do lar, os recursos financeiros, a disponibilidade e qualidade de cuidados de saúde e sociais, as oportunidades de adquirir novas informações e habilidades, as atividades de lazer, o ambiente físico e $\mathrm{o}$ transporte ${ }^{18}$.

Todos os participantes foram previamente informados sobre os objetivos da pesquisa e convidados a assinar o Termo de Consentimento Livre e Esclarecido, garantindo o sigilo e o anonimato dos envolvidos.

Os dados foram tabulados e exportados para o software Statistical Package for the Social Sciences (SPSS) v. 17.0 (IBM, Armonk, NY, EUA), sendo as análises realizadas considerando um nível de confiança de $95 \%$ e significância com $\mathrm{p}<0,05$. Os escores obtidos foram transformados em uma escala linear que variou de 0-100, sendo estes, respectivamente, os valores menos e mais favoráveis de QV, conforme sintaxe proposta pelo WHOQOL group $^{18}$. Para a análise dos escores de QV, utilizou-se uma escala adaptada ${ }^{19}$, sendo categorizada da seguinte maneira: valores entre $0 \mathrm{e}$ 40 eram considerados região de "insatisfação"; de 41 a 69, corresponderam à região de "indefinição"; e, acima de 70, como sendo a região de "satisfação". Para efeito da análise estatística, as variáveis foram compiladas em dois grupos, pois apenas 1 aluno foi classificado na categoria indefinição: "insatisfação/indefinição" e "satisfação". Os escores abaixo de 70 foram considerados como "insatisfação/indefinição" com a QV e aqueles acima desse valor como "satisfação".

Os valores dos escores de cada domínio foram submetidos ao teste de KolmogorovSmirnov, para que estes fossem padronizados e observada a distribuição normal dos dados. Estes 
foram analisados pelo teste ANOVA, para medidas repetidas, seguido do pós-teste de Bonferronni, para avaliação da diferença entre os domínios aferidos. Os dados foram descritos nas frequências absoluta e percentual, e analisados por meio do teste do qui-quadrado $\left(\chi^{2}\right)$. Foi também utilizado o modelo de regressão logística multinomial, a fim de verificar a relação de causalidade entre a categoria de QV e os dados sociodemográficos investigados.

O estudo foi aprovado pelo Comitê de Ética e Pesquisa da UFC, sob o parecer de $\mathrm{n}^{\mathrm{o}}$ 1.113.226.

\section{RESULTADOS}

Cerca de 55,7\% dos discentes foram classificados na região de satisfação quanto à QV; $61,4 \%$ eram do sexo feminino, metade eram casados e $73,9 \%$ relataram não possuir filhos; $34,1 \%$ dos discentes relataram dedicar-se exclusivamente às atividades da pós-graduação; e $59,1 \%$ possuíam renda mensal de mais de quatro salários mínimos. A prática religiosa foi afirmada por $89,8 \%$ e $75,0 \%$ consideraram-se religiosos. Possuíam bolsa na pós-graduação $68,2 \%$ dos participantes. Cursavam o doutorado ou pósdoutorado 53,4\% dos estudantes, enquanto 46,6\% encontravam-se no segundo ano do curso da pósgraduação. Foram inseridos os alunos de pósdoutorado pois o intuito do trabalho era abranger todos os discentes de pós-graduação, e estes foram agrupados aos alunos de doutorado pois representavam uma pequena porcentagem dos discentes (3,40\%), sendo apenas 3 alunos.

O domínio Físico retratou a menor média $(12,82 \pm 1,56)$, apresentando significância ( $<<0,001)$ quando comparado aos demais domínios. O domínio Relações Sociais apresentou a maior média $(15,23 \pm 2,72)$ (tabela 1 ).

Tabela 1. Média individual dos domínios do WHOQOL-bref

\begin{tabular}{lllllll}
\hline Domínios & Média & DP & Mediana & Mínima & Máxima & p-Valor \\
\hline Físico* & 12,82 & 1,56 & 13,14 & 8,57 & 16,57 & $<0,001$ \\
Psicológico & 14,12 & 1,48 & 14,00 & 11,33 & 18,00 & \\
Relações Sociais & 15,23 & 2,72 & 15,33 & 8,00 & 20,00 & \\
Meio Ambiente & 14,35 & 1,64 & 14,50 & 10,50 & $18 ., 00$ & \\
\hline
\end{tabular}

*p $<0,05$ versus todos os grupos. Teste ANOVA/Bonferronni. DP: Desvio Padrão.

A tabela 2 retrata a associação entre os dados sociodemográficos e o domínio Físico. Observouse significância ao associar a frequência da prática religiosa e ano da pós-graduação com o domínio Físico. A frequência "sempre" dessa prática foi significativamente associada à região de insatisfação/indefinição $\quad(\mathrm{p}=0,046)$; enquanto a classificação "às vezes" foi significativamente associada à região de satisfação $(\mathrm{p}=0,046)$ (IC 95\%: 1,1 - 77,9). Também houve associação significativa entre os alunos do terceiro ano da pósgraduação com a classificação satisfação nesse domínio (p=0,025) (IC 95\%: 1,8 - 6310,3).
A tabela 3 mostra a associação entre os dados sociodemográficos e o domínio Psicológico. O sexo feminino mostrou associação significativa com insatisfação/indefinição no referido domínio ( $\mathrm{p}=0,016)$; já o masculino demonstrou associação significativa com satisfação nesse domínio $(\mathrm{p}=0,016)$ (IC 95\%: 1,4 - 28,9). Considerar-se religioso foi significativamente associado à insatisfação/indefinição nesse domínio $(\mathrm{p}=0,008)$, enquanto não se considerar religioso foi significativamente associado à região de satisfação com os aspectos desse domínio ( $\mathrm{p}=0,008)$ (IC 95\%: $2,1-177,1)$. 
Tabela 2. Associação entre os dados sociodemográficos e o domínio Físico

\begin{tabular}{|c|c|c|c|c|}
\hline \multirow[b]{2}{*}{ Variáveis } & \multicolumn{2}{|l|}{ Domínio Físico } & \multirow[b]{2}{*}{ p-Valor* } & \multirow[b]{2}{*}{ p-Valor ${ }^{\dagger}$} \\
\hline & $\begin{array}{c}\text { Insatisfação/Indefinição } \\
\text { n (\%) }\end{array}$ & $\begin{array}{l}\text { Satisfação } \\
\text { n (\%) }\end{array}$ & & \\
\hline \multicolumn{5}{|c|}{ 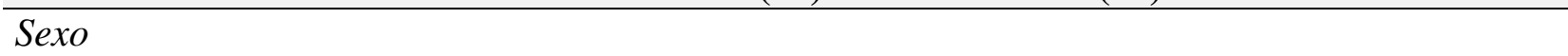 } \\
\hline Masculino & $25(36,8)$ & $9(45,0)$ & \multirow[t]{2}{*}{0,506} & \\
\hline Feminino & $43(63,2)$ & $11(55,0)$ & & \\
\hline \multicolumn{5}{|l|}{ Estado civil } \\
\hline Solteiro & $37(54,4)$ & $6(30,0)$ & \multirow{2}{*}{0,055} & \\
\hline Casado/Divorciado & $31(45,6)$ & $14(70,0)$ & & \\
\hline \multicolumn{5}{|l|}{ Filhos } \\
\hline Sim & $14(20,6)$ & $9 *(45,0)$ & \multirow[t]{2}{*}{0,029} & \\
\hline Não & $54 *(79,4)$ & $11(55,0)$ & & \\
\hline \multicolumn{5}{|c|}{ Trabalho - Dedicação exclusiva } \\
\hline Não & $45(66,2)$ & $13(65,0)$ & \multirow[t]{2}{*}{0,922} & \\
\hline Sim & $23(33,8)$ & $7(35,0)$ & & \\
\hline \multicolumn{5}{|l|}{ Trabalho - Cirurgião-dentista } \\
\hline Não & $32(47,1)$ & $9(45,0)$ & \multirow{2}{*}{0,871} & \\
\hline Sim & $36(52,9)$ & $11(55,0)$ & & \\
\hline \multicolumn{5}{|l|}{ Renda Mensal } \\
\hline Até 2 SM & $13(19,1)$ & $2(10,0)$ & \multirow[t]{3}{*}{0,093} & \\
\hline 2 a 4 SM & $19(27,9)$ & $2(10,0)$ & & \\
\hline$>4 \mathrm{SM}$ & $36(53)$ & $16(80,0)$ & & \\
\hline \multicolumn{5}{|l|}{ Prática de alguma religião } \\
\hline Sim & $62(91,2)$ & $17(85,0)$ & \multirow[t]{2}{*}{0,423} & \\
\hline Não & $6(8,8)$ & $3(15,0)$ & & \\
\hline \multicolumn{5}{|c|}{ Frequência da prática religiosa } \\
\hline Nunca & $5(7,4)$ & $2(10,0)$ & \multirow[t]{4}{*}{0,431} & 0,046 \\
\hline Raramente & $6(8,8)$ & $4(20,0)$ & & \\
\hline Às vezes & $30(44,1)$ & $9 \dagger(45,0)$ & & \\
\hline Sempre & $27 \dagger(39,7)$ & $5(25,0)$ & & \\
\hline \multicolumn{5}{|l|}{ Considera-se religioso } \\
\hline Sim & $52(76,5)$ & $14(70,0)$ & \multirow[t]{2}{*}{0,557} & \\
\hline Não & $16(23,5)$ & $6(30,0)$ & & \\
\hline \multicolumn{5}{|l|}{ Bolsista } \\
\hline Sim & $47(69,1)$ & $13(65,0)$ & \multirow[t]{2}{*}{0,728} & \\
\hline Não & $21(30,9)$ & $7(35,0)$ & & \\
\hline \multicolumn{5}{|l|}{ Curso na pós-graduação } \\
\hline Mestrado & $34(50)$ & $7(35,0)$ & 0,237 & \\
\hline Doutorado/Pós-doutorado & $34(50)$ & $13(65,0)$ & & \\
\hline Ano na pós-graduação & & & & \\
\hline Primeiro & $23(33,8)$ & $6(30,0)$ & 0,016 & 0,025 \\
\hline Segundo & $35(51,4)$ & $6(30,0)$ & & \\
\hline Terceiro & $5(7,4)$ & $7 * \dagger(35,0)$ & & \\
\hline Quarto & $5(7,4)$ & $1(5,0)$ & & \\
\hline
\end{tabular}


Tabela 3. Associação entre os dados sociodemográficos e o domínio Psicológico

\begin{tabular}{|c|c|c|c|c|}
\hline \multirow[b]{2}{*}{ Variáveis } & \multicolumn{2}{|c|}{ Domínio Psicológico } & \multirow[b]{2}{*}{ p-Valor* } & \multirow[b]{2}{*}{ p-Valor ${ }^{\dagger}$} \\
\hline & $\begin{array}{c}\text { Insatisfação/ Indefinição } \\
\text { n (\%) }\end{array}$ & $\begin{array}{l}\text { Satisfação } \\
\text { N (\%) }\end{array}$ & & \\
\hline \multicolumn{5}{|l|}{ Sexo } \\
\hline Masculino & $14(29,2)$ & $20 * \dagger(50,0)$ & 0,046 & 0,016 \\
\hline Feminino & $34 * \dagger(70,8)$ & $20(50,0)$ & & \\
\hline \multicolumn{5}{|l|}{ Estado civil } \\
\hline Solteiro & $26(54,2)$ & $17(42,5)$ & 0,276 & \\
\hline Casado/Divorciado & $22(45,8)$ & $23(57,5)$ & & \\
\hline \multicolumn{5}{|l|}{ Filhos } \\
\hline Sim & $12(25,0)$ & $11(27,5)$ & 0,790 & \\
\hline Não & $36(75,0)$ & $29(72,5)$ & & \\
\hline \multicolumn{5}{|c|}{ Trabalho - Dedicação exclusiva } \\
\hline Não & $30(62,5)$ & $28(70,0)$ & 0,460 & \\
\hline Sim & $18(37,5)$ & $12(30,0)$ & & \\
\hline \multicolumn{5}{|l|}{ Trabalho - Cirurgião-dentista } \\
\hline Não & $23(47,9)$ & $18(45)$ & 0,785 & \\
\hline Sim & $25(52,1)$ & $22(55 \%)$ & & \\
\hline \multicolumn{5}{|l|}{ Renda Mensal } \\
\hline Até 2 SM & $10(20,8)$ & $5(12,5)$ & 0,585 & \\
\hline 2 a 4 SM & $11(22,9)$ & $10(25,0)$ & & \\
\hline$>4 \mathrm{SM}$ & $27(56,3)$ & $25(62,5)$ & & \\
\hline \multicolumn{5}{|l|}{ Prática de alguma religião } \\
\hline Sim & $42(87,5)$ & $37(92,5)$ & 0,441 & \\
\hline Não & $6(12,5)$ & $3(7,5)$ & & \\
\hline \multicolumn{5}{|l|}{ Frequência da prática religiosa } \\
\hline Nunca & $5(10,4)$ & $2(5,0)$ & 0,820 & \\
\hline Raramente & $5(10,4)$ & $5(12,5)$ & & \\
\hline Às vezes & $21(43,8)$ & $18(45,0)$ & & \\
\hline Sempre & $17(35,4)$ & $15(37,5)$ & & \\
\hline \multicolumn{5}{|l|}{ Considera-se religioso } \\
\hline Sim & $39 \dagger(81,2)$ & $27(67,5)$ & 0,138 & 0,008 \\
\hline Não & $9(18,8)$ & $13+(32,5)$ & & \\
\hline \multicolumn{5}{|l|}{ Bolsista } \\
\hline Sim & $31(64,6)$ & $29(72,5)$ & 0,427 & \\
\hline Não & $17(35,4)$ & $11(27,5)$ & & \\
\hline \multicolumn{5}{|l|}{ Curso na pós-graduação } \\
\hline Mestrado & $22(45,8)$ & $19(47,5)$ & 0,876 & \\
\hline Doutorado/Pós-doutorado & $26(54,2)$ & $21(52,5)$ & & \\
\hline \multicolumn{5}{|l|}{ Ano na pós-graduação } \\
\hline Primeiro & $19(39,6)$ & $10(25,0)$ & 0,021 & \\
\hline Segundo & $19(39,6)$ & $22 *(55,0)$ & & \\
\hline Terceiro & $4(8,3)$ & $8 *(20,0)$ & & \\
\hline Quarto & $6 *(12,5)$ & - & & \\
\hline
\end{tabular}


A associação entre os dados sociodemográficos e os domínios Relações Sociais e Meio Ambiente foram retratados nas tabelas $4 \mathrm{e}$ 5 , respectivamente. O estado civil solteiro mostrouse significativamente associado com a categoria insatisfação/indiferença, tanto no domínio Relações Sociais $(p=0,017)$, quanto do Meio Ambiente ( $\mathrm{p}=0,008)$. A variável estado civil casado/divorciado foi significativamente associada com a categoria satisfação no domínio Relações Sociais $(\mathrm{p}=0,017)$ e Meio Ambiente $(\mathrm{p}=0,008)$.

\section{DISCUSSÃO}

Os alunos da pós-graduação em Odontologia foram classificados no grupo de satisfação quanto à QV. Considerando a subjetividade na definição de QV, e que esta tem relação direta com a autoestima, tal resultado corrobora com o estudo realizado com estudantes de pós-graduação de uma universidade do sul do Brasil, que verificou uma elevada autoestima nessa população ${ }^{20}$. Porém, em estudo realizado com alunos de pós-graduação na Universidade Federal do Rio de Janeiro, os autores demonstraram preocupação com a QV dos estudantes, pois observaram um alto índice de estresse entre eles ${ }^{21}$. Embora sejam o mesmo tipo de população, a autoavaliação de QV leva em consideração critérios subjetivos e pessoais, que diferem entre os indivíduos ${ }^{1}$, o que pode explicar a diferença de resultados entre as pesquisas citadas quanto a este quesito.

Dentre os 88 alunos envolvidos na pesquisa, a maior média se deu no domínio Relações Sociais e a menor no Físico, semelhante ao encontrado em estudo que avaliou a QV dos alunos de graduação e pós-graduação em Odontologia em São Paulo ${ }^{13}$. Tais resultados diferem de pesquisa realizada com estudantes de pós-graduação em Odontologia dos Estados Unidos ${ }^{12}$, adultos chineses $^{22}$ e com iranianas adultas ${ }^{23}$, sendo nestes estudos o domínio Físico o de maior escore. Já estudo realizado com alunos de pós-graduação em Odontologia no
Ceará, durante o isolamento social na pandemia de COVID-19, observou que o domínio Psicológico foi o de menor média ${ }^{14}$. O menor escore no domínio Físico foi também o encontrado no estudo com mestrandos e doutorandos brasileiros, e se explica tal resultado possivelmente pela extensa carga-horária exigida pela pós-graduação stricto sensu, tendo o aluno que conciliá-la com as demais atividades do seu dia-a-dia, como a sua vida pessoal e o emprego ${ }^{6}$, fatores que podem justificar os resultados encontrados do domínio Físico nesse estudo. Quanto ao domínio Relações Sociais, o resultado encontrado provavelmente se deu pelo fato de todos os alunos serem adultos e cirurgiõesdentistas formados, já possuindo suas relações sociais consolidadas.

A relação inversa entre a frequência da prática religiosa e o domínio Físico do presente trabalho foi diferente do encontrado num estudo que investigou a relação entre religiosidade e QV em pessoas com vírus da imunodeficiência humana ${ }^{24}$. Nele, a relação entre religiosidade se deu com os domínios Psicológico, Relações Sociais e Meio Ambiente ${ }^{24}$. Esse resultado pode ser justificado pelo fato da religiosidade desempenhar um papel de favorecedora de bem-estar psicológico e de apoio social às pessoas acometidas por doenças e outras manifestações clínicas, conforme afirmam outras pesquisas ${ }^{25-27}$, o que pode impactar em aspectos do domínio Físico, como dor e desconforto, energia e fadiga. Nesse sentido, os indivíduos podem estar em busca de melhora desses sintomas por meio de práticas espirituais. Por se tratar de estudo transversal, não se pode estabelecer relação de causalidade. Porém, aparentemente, a procura pela religiosidade pelos discentes aconteceu em momentos de maior desconforto físico, estando relacionada à maior insatisfação quanto à QV .

No domínio Físico foi observada, ainda, significância estatística entre o terceiro ano da pósgraduação e a satisfação quanto à QV. Este resultado 
Tabela 4. Associação entre os dados sociodemográficos e o domínio Relações Sociais

\begin{tabular}{|c|c|c|c|c|}
\hline \multirow[b]{2}{*}{ Variáveis } & \multicolumn{2}{|c|}{ Relações Sociais } & \multirow[b]{2}{*}{ p-Valor* } & \multirow[b]{2}{*}{ p-Valor ${ }^{\dagger}$} \\
\hline & $\begin{array}{c}\text { Insatisfação/Indefiniçãão } \\
\text { n (\%) }\end{array}$ & $\begin{array}{l}\text { Satisfação } \\
\text { n }(\%)\end{array}$ & & \\
\hline \multicolumn{5}{|c|}{$y^{\prime}$} \\
\hline Masculino & $9(37,5)$ & $25(39,1)$ & 0,893 & \\
\hline Feminino & $15(62,5)$ & $39(60,9)$ & & \\
\hline \multicolumn{5}{|l|}{ Estado civil } \\
\hline Solteiro & $15 \dagger(62,5)$ & $28(43,7)$ & 0,117 & 0,017 \\
\hline Casado/Divorciado & $9(37,5)$ & $36 \dagger(56,3)$ & & \\
\hline \multicolumn{5}{|l|}{ Filhos } \\
\hline Sim & $7(29,2)$ & $16(25,0)$ & 0,692 & \\
\hline Não & $17(70,8)$ & $48(75,0)$ & & \\
\hline \multicolumn{5}{|c|}{ Trabalho - Dedicacão exclusiva } \\
\hline Não & $17(70,8)$ & $41(64,1)$ & 0,551 & \\
\hline Sim & $7(29,2)$ & $23(35,9)$ & & \\
\hline \multicolumn{5}{|l|}{ Trabalho - Cirurgião-dentista } \\
\hline Não & $12(50,0)$ & $29(45,3)$ & 0,695 & \\
\hline Sim & $12(50,0)$ & $35(54,7)$ & & \\
\hline \multicolumn{5}{|l|}{ Renda Mensal } \\
\hline Até 2 SM & $4(16,7)$ & $11(17,2)$ & 0,907 & \\
\hline 2 a 4 SM & $5(20,8)$ & $16(25,0)$ & & \\
\hline$>4 \mathrm{SM}$ & $15(62,5)$ & $37(57,8)$ & & \\
\hline \multicolumn{5}{|l|}{ Prática de alguma religião } \\
\hline Sim & $23(95,8)$ & $56(87,5)$ & 0,251 & \\
\hline Não & $1(4,2)$ & $8(12,5)$ & & \\
\hline \multicolumn{5}{|c|}{ Frequência da prática religiosa } \\
\hline Nunca & $1(4,1)$ & $6(9,4)$ & 0,547 & \\
\hline Raramente & $4(16,7)$ & $6(9,4)$ & & \\
\hline Às vezes & $12(50,0)$ & $27(42,1)$ & & \\
\hline Sempre & $7(29,2)$ & $25(39,1)$ & & \\
\hline \multicolumn{5}{|l|}{ Bolsista } \\
\hline Sim & $15(62,5)$ & $45(70,3)$ & 0,483 & \\
\hline Não & $9(37,5)$ & $19(29,7)$ & & \\
\hline \multicolumn{5}{|l|}{ Curso na pós-graduação } \\
\hline Mestrado & $8(33,3)$ & $33(51,6)$ & 0,127 & \\
\hline Doutorado/Pós-doutorado & $16(66,7)$ & $31(48,4)$ & & \\
\hline \multicolumn{5}{|l|}{ Ano na pós-graduação } \\
\hline Primeiro & $8(33,3)$ & $21(32,8)$ & 0,917 & \\
\hline Segundo & $10(41,7)$ & $31(48,4)$ & & \\
\hline Terceiro & $4(16,7)$ & $8(12,5)$ & & \\
\hline Quarto & $2(8,3)$ & $4(6,3)$ & & \\
\hline
\end{tabular}


Tabela 5. Associação entre os dados sociodemográficos e o domínio Meio Ambiente

\begin{tabular}{|c|c|c|c|c|}
\hline \multirow[b]{2}{*}{ Variáveis } & \multicolumn{2}{|l|}{ Meio Ambiente } & \multirow[b]{2}{*}{ p-Valor* } & \multirow[b]{2}{*}{ p-Valor ${ }^{\dagger}$} \\
\hline & $\begin{array}{c}\text { Insatisfação/Indefinição } \\
\text { n (\%) }\end{array}$ & $\begin{array}{c}\text { Satisfação } \\
\text { n }(\%)\end{array}$ & & \\
\hline \multicolumn{5}{|c|}{ ( } \\
\hline Masculino & $14(32,6)$ & $20(44,4)$ & 0,252 & \\
\hline Feminino & $29(67,4)$ & $25(55,6)$ & & \\
\hline \multicolumn{5}{|l|}{ Estado civil } \\
\hline Solteiro & $28 * \dagger(65,1)$ & $15(33,3)$ & 0,003 & 0,008 \\
\hline Casado/Divorciado & $15(34,9)$ & $30 * \dagger(66,7)$ & & \\
\hline \multicolumn{5}{|l|}{ Filhos } \\
\hline Sim & $9(20,9)$ & $14(31,1)$ & 0,277 & \\
\hline Não & $34(79,1)$ & $31(68,9)$ & & \\
\hline \multicolumn{5}{|c|}{ Trabalho - Dedicação exclusiva } \\
\hline Não & $25(58,1)$ & $33(73,3)$ & 0,133 & \\
\hline Sim & $18(41,9)$ & $12(26,7)$ & & \\
\hline \multicolumn{5}{|l|}{ Trabalho - Cirurgião-dentista } \\
\hline Não & $23(53 ., 5)$ & $18(40,0)$ & 0,205 & \\
\hline Sim & $20(46,5)$ & $27(60,0)$ & & \\
\hline \multicolumn{5}{|l|}{ Renda Mensal } \\
\hline Até $2 \mathrm{SM}$ & $11(25,6)$ & $4(8,9)$ & 0,040 & \\
\hline 2 a $4 \mathrm{SM}$ & $12(27, .9)$ & $9(20,0)$ & & \\
\hline$>4 \mathrm{SM}$ & $20(46,5)$ & $32(71,1)$ & & \\
\hline \multicolumn{5}{|l|}{ Prática de alguma religião } \\
\hline Sim & $40(93,0)$ & $39(86,7)$ & 0,325 & \\
\hline Não & $3(7,0)$ & $6(13,3)$ & & \\
\hline \multicolumn{5}{|c|}{ Frequência da prática religiosa } \\
\hline Nunca & $2(4,6)$ & $5(11,1)$ & 0,497 & \\
\hline Raramente & $5(11,6)$ & $5(11,1)$ & & \\
\hline Às vezes & $22(51,2)$ & $17(37,8)$ & & \\
\hline Sempre & $14(32,6)$ & $18(40,0)$ & & \\
\hline \multicolumn{5}{|l|}{ Bolsista } \\
\hline Sim & $30(69,8)$ & $30(66,7)$ & 0,755 & \\
\hline Não & $13(30,2)$ & $15(33,3)$ & & \\
\hline \multicolumn{5}{|l|}{ Curso na pós-graduação } \\
\hline Mestrado & $24(55,8)$ & $17(37,8)$ & 0,090 & \\
\hline Doutorado/Pós-doutorado & $19(44,2)$ & $28(62,2)$ & & \\
\hline \multicolumn{5}{|l|}{ Ano na pós-graduação } \\
\hline Primeiro & $15(34,8)$ & $14(31,1)$ & 0,530 & \\
\hline Segundo & $22(51,2)$ & $19(42,2)$ & & \\
\hline Terceiro & $4(9,3)$ & $8(17,8)$ & & \\
\hline Quarto & $2(4,7)$ & $4(8,9)$ & & \\
\hline
\end{tabular}

*Teste $\chi^{2}\left({ }^{*} \mathrm{p}<0,05\right) ;{ }^{\dagger}$ Regressão Logística Multinomial $(\dagger \mathrm{p}<0,05)$. As caselas em branco indicam ausência de associação significativa. SM - Salário mínimo (R \$788,00). 
difere do encontrado em uma pesquisa realizada com os estudantes de pós-graduação em ciências da saúde do The American College Health Association's National College Health Assessment $(\mathrm{ACHA}-\mathrm{NCHA})^{28}$, que relacionou o estresse de doutorandos ao ambiente da pós-graduação. O achado desse estudo pode estar relacionado com a temporaneidade da coleta de dados, visto que a pesquisa foi transversal, não levando em consideração a relação das atividades com o período de todos os semestres da pós-graduação.

A maioria das mulheres foi classificada na região de insatisfação/indefinição quanto aos itens abordados no domínio Psicológico, apresentando resultado semelhante a estudo que avaliou o estresse de pós-graduandos da UFRJ, sendo constatada associação entre o sexo feminino e o estresse $^{21}$. Ainda, em estudo realizado com estudantes de pós-graduação de todo o Brasil, o maior nível de estresse também se deu no sexo feminino $^{6}$. Esse achado também pode ser justificado pelas inúmeras atividades acumuladas pelas mulheres, como os afazeres domésticos, o cuidar da família, as atividades laborais, e as atividades da pós-graduação.

Dentre os aspectos abordados no domínio Psicológico estão os sentimentos positivos e negativos e a espiritualidade. $\mathrm{O}$ resultado encontrado, no qual a maioria dos alunos que se consideravam religiosos foi classificada na região de insatisfação/indiferença quanto aos aspetos abordados nesse domínio, pode ser explicado pela relação entre religião, espiritualidade e crenças pessoais, e QV em pessoas com a saúde comprometida $^{26,29}$, sendo a pós-graduação stricto sensu um programa de estudo que exige em demasia dos alunos, podendo gerar, inclusive, preocupações psicossociais $^{28}$.

A associação entre o estado civil e os domínios Relações Sociais e Meio Ambiente pode ser fundamentada pelos aspectos abordados em ambos. O suporte social e atividade sexual relacionam-se com o domínio Relações Sociais, enquanto o ambiente do lar e os recursos financeiros com o Meio Ambiente. Os indivíduos casados geralmente possuem esses elementos mais ajustados. Tais achados corroboram com o estudo que objetivou investigar a $\mathrm{QV}$ por sexo em pacientes com esquizofrenia, explorando o domínio ocupacional, no qual o estado civil apresentou-se como a variável mais relevante, e mulheres e homens solteiros apresentaram baixa QV quando comparados com os casados ${ }^{30}$.

Pesquisas nas quais o WHOQOL-bref tenha sido aplicado a estudantes de programas pósgraduação stricto sensu em Odontologia não são frequentes na literatura, incluindo a análise de cada domínio com esse público, o que torna o presente estudo relevante. No entanto, este fato limita a comparação dos resultados. A representatividade de $100 \%$ do universo pesquisado, a presença de estudantes em todos os anos da pós-graduação e o uso de um instrumento transcultural validado no Brasil para aferição da QV também são aspectos relevantes deste estudo.

Os resultados do presente estudo devem ser interpretados à luz das limitações de seu desenho transversal, que não permite estabelecer a precedência temporal sobre os desfechos avaliados, assim como da não representatividade para alunos matriculados em outros programas de pósgraduação em Odontologia.

Investigações futuras que envolvam outros aspectos relacionando estudantes de pós-graduação stricto sensu em Odontologia e QV, como um estudo do tipo longitudinal, e que analise as questões elencadas em cada domínio, para investigações em diferentes períodos da pósgraduação, ou estudos comparativos entre instituições diferentes, são necessários.

\section{CONCLUSÕES}

A QV dos estudantes de pós-graduação em Odontologia foi mensurada na região de satisfação, 
podendo ser influenciada por fatores como religiosidade, ano da pós-graduação, sexo e estado civil. Sugere-se um olhar criterioso de toda a rede de apoio que envolve os alunos de pós-graduação em Odontologia, como a família, o curso em si, além da atividades laborais destes, possibilitando um maior suporte ao estudante, para que o período do curso de pós-graduação stricto sensu transcorra com mais QV.

\section{ABSTRACT \\ Quality of life of postgraduate students in Dentistry: an analysis through the WHOQOL- bref domains}

The objective of the study was to assess the quality of life (QoL) of postgraduate students in Dentistry through the domains of the World Health Organization Questionnaire for Quality of Life-bref (WHOQOL-bref). This is a crosssectional study, with 88 students enrolled in a postgraduate program in Dentistry. Data were collected between June and August 2015, including sociodemographic information and a quality of life questionnaire, the WHOQOL-bref. Sociodemographic information was associated with the domains of the questionnaire used. Bivariate analysis, followed by the multinomial logistic regression model were carried out, with $\mathrm{p}<0.05$ being considered significant. The majority $(55.7 \%)$ of students were classified in the "satisfaction" category in terms of quality of life. In the domains, the highest score found was in Social Relations $(15.23 \pm 2.72)$, and the lowest, in Physical (12.82 \pm 1.56$)$. There was an association between the frequency of religious practice $(\mathrm{p}=0.046)$ and the year of postgraduate school $(p=0.025)$ with the Physical domain;

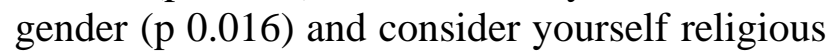
$(p=0.008)$ with the Psychological domain; and marital status with the Social Relations $(\mathrm{p}=0.017)$ and Environment $(\mathrm{p}=0.008)$ domains. The QoL of postgraduate students in Dentistry was measured in the region of satisfaction, which can be influenced by factors such as religiosity, year of graduation, gender and marital status.

Descriptors: Quality of Life. Dentistry.
Education, Dental, Graduate.

\section{REFERÊNCIAS}

1. The World Health Organization Quality of Life Assessment (WHOQOL): position paper from the World Health Organization. Soc Sci Med. 1995; 41(10): 1403-9.

2. Rodrigues MIQ, Frota LMA, Frota MMA, Teixeira CNG. Stress factors and quality of life of Dental students. Rev ABENO. 2019; 19(1): 49-57.

3. Lima RS, Cavalcante JL, Machado MFAS, Cavalcante EGR, Quirino GS, Rebouças VCF. Qualidade de vida de acadêmicos de enfermagem: análise à luz do modelo de pender. Rev Rene. 2020; 21.

4. Vadeboncoeur C, Townsend N, Foster C. A meta-analysis of weight gain in first year university students: is freshman 15 a myth? BMC Obes. 2015; 2: 22.

5. Macedo E, Souza CP. A pesquisa em educação no Brasil. Rev Bras Educ. 2010; 15(43): 166-76.

6. Faro A. Estresse e Estressores na PósGraduação: Estudo com Mestrandos e Doutorandos no Brasil. Psicol Teor Pesqui. 2013; 29(1): 51-60.

7. Nagy GA, Fang CM, Hish AJ, Kelly L, Nicchitta CV, Dziarasa K, et al. Problemas de Burnout e Saúde Mental em Doutorandos Biomédicos. CBE Life Sci Educ. 2019; 18(2):27.

8. Brasil. Ministério da Ciência e Tecnologia. Plano de ação em ciência, tecnologia e inovação: Principais resultados e avanços [Internet]. Brasília (DF): Ministério da Ciência e Tecnologia; 2010 [Acesso em 21 Maio 2019]. Disponível em: http://protec.org.br/uploads/paginas/file/publ icacoes/Balan\%C3\%A7oPACTI.PDF.

9. McKenna L, Robinson E, Penman J, Hills D. Factors impacting on psychological 
wellbeing of international students in the health professions: A scoping review. Int J Nurs Stud. 2017; 74: 85-94.

10. Bazrafkan L, Shokrpour N, Yousefi A, Yamani N. Management of stress and anxiety among PhD students during thesis writing: a qualitative study. Health Care Manag. 2016; 35(3): 231-40.

11. Bhandari P. Stress and health related quality of life of Nepalese students studying in South Korea: a cross sectional study. Health Qual Life Outcomes. 2012; 10(1):26.

12. Andre A, Pierre GC, McAndrew M. Quality of life among dental students: a survey study. JDE 2017; 81(10): 1164-70.

13. Amadeu JR, Justi MM. Qualidade de vida de estudantes de graduação e pós-graduação em Odontologia. Arch Health Invest. 2017; 6(11): 540-4.

14. Silva PGB, Oliveira CAL, Borges MMF, Moreira DM, Alencar PNB, Avelar RL, et al. Distance learning during social seclusion by COVID-19: improving the quality of life of undergraduate dentistry students. Eur J Dent Educ. 2021;25:124-34.

15. Fleck MP, Louzada S, Xavier M, Chachamovich E, Vieira G, Santos L, et al. Aplicação da versão em português do instrumento abreviado de avaliação da qualidade de vida "WHOQOL-Bref". Rev Saúde Pública. 2000; 34(2): 178-83.

16. Rouquayrol MZ, Filho NA. Epidemiologia \& Saúde. Rio de Janeiro: Guanabara Koogan; 2003, 736p.

17. The World Health Organization Quality of Life Assessment (WHOQOL): development and general psychometric properties. Soc Sci Med. 1998; 46(12):1569-85.

18. Whoqol. The Whoqol Group. Sintaxe SPSS WHOQOL - bref Questionnaire. [serial on the internet] [Acesso em 20 Set 2018]. Disponível em: https://www.ufrgs.br/ qualidep/ qualidade-de-vida/projeto-whoqolbref.

19. Brito DP, Oliveira LMR, Braga SR, Nuto SAS, Viana FAC. Avaliação da qualidade de vida de acadêmicos de Odontologia do estado do Ceará. Coleç Pesqui Educ Fís. 2012; 11(3):41-50.

20. Silva AH, Vieira KM. Sindrome de Bornout em estudantes de Pós-graduação: uma análise da influência da autoestima e relação orientador-orientando. Pretexto. 2015; 16(1): 52-68.

21. Malagris LE, Suassuna AT, Bezerra DV, Hirata HP, Monteiro JL, Silva LR, et al. Níveis de estresse e características sociobiográficas de alunos de pós-graduação. Psicol Rev. 2009; 15(1):184-203.

22. Deng Q, Wang LM, Zhang M. Quality of life and related influencing factors in Chinese adults. Zhonghua Liu Xing Bing Xue Za Zhi. 2016; 37(2): 243-7.

23. Salehi A, Harris N, Sebar B, Coyne E. The relationship between living environment, well-being and lifestyle behaviours in young women in Shiraz, Iran. Health Soc Care Community. 2017; 25(1): 275-84.

24. Medeiros B, Saldanha AA. Religiosidade e qualidade de vida em pessoas com HIV. Estud Psicol. 2012; 29(1): 53-61.

25. Ai AL, Tice TN, Huang B, Rodgers W, Bolling SF. Types of prayer, optimism, and well-being of middle-aged and older patients undergoing open-heart surgery. Ment Health, Relig Cult. 2008; 11(1):131-50.

26. Fleck MP, Borges ZN, Bolognesi G, Rocha NS. Desenvolvimento do WHOQOL, módulo espiritualidade, religiosidade e crenças pessoais. Rev Saúde Pública. 2003; 37(4): 446-55.

27. Moreira-Almeida A, Lotufo Neto F, Koenig HG. Religiousness and mental health: a review. Rev Bras Psiquiatr. 2006; 28(3): 242- 
50.

28. Kernan W, Bogart J, Wheat ME. Health related barriers to learning among graduate students. Health Educ. 2011; 111(5): 425-45.

29. WHOQOL SRPB Group. A cross-cultural study of spirituality, religion, and personal beliefs as components of quality of life. Soc Sci Med. 2006; 62(6): 1486-97.
30. Cardoso CS, Caiaffa WT, Bandeira M, Siqueira AL, Abreu MN, Fonseca JO. Qualidade de vida e dimensão ocupacional na esquizofrenia: uma comparação por sexo. Cad Saúde Pública. 2006; 22(6): 1303-14.

\section{Correspondência para:}

Cinthia Nara Gadelha Teixeira

e-mail: cinthia_n80@yahoo.com.br

Rua Jóckey Clube, 48 - altos.

Bairro Jóckey Clube

60510-115 Fortaleza/CE 